\title{
ANÁLISE DA PRESENÇA DE MICRORGANISMOS DO INTERIOR DE MALETAS TRANSPORTADAS POR ALUNOS DE GRADUAÇÃO
}

\section{ANALYSIS OF THE PRESENCE OF MICROORGANISMS WITHIN SUITCASES CARRIED BY UNDERGRADUATE STUDENTS}

\author{
Miguel Simão Haddad Filho* \\ Thais Pereira Leal ${ }^{*}$ \\ Luiz Fernando Ferrari Belasalma*** \\ José Eduardo Bacci"*** \\ Maria Teresa Botti Rodrigues dos Santos ${ }^{* \cdots * *}$ \\ João Marcelo Ferreira de Medeiros"*n+*
}

\begin{abstract}
RESUMO
Introdução: Muito se tem aprimorado no sentido de oferecer maior nível de segurança aos nossos pacientes e a toda equipe promotora da saúde, à custa de protocolos de biossegurança, cada vez mais amplos, rígidos e confiáveis, para tal, um arsenal de recursos é empregado para maximizar o controle microbiano. Nesse sentido, os cursos de odontologia aplicam altos investimentos em sistemas que possibilitem tal controle; a assertiva continuada do conhecimento, treinamento que coloque em prática as barreiras de proteção, que permite oferecer atendimento livre de riscos e, consequentemente, desenvolver responsabilidade na prevenção das doenças em todos os níveis de atenção, sob o monitoramento atento dessas atitudes por parte dos educadores. Entretanto, ainda se percebe negligência quanto ao material de consumo acondicionado em maletas plásticas com divisórias, transportada pelo aluno. Este estudo teve por objetivo pesquisar os microrganismos presentes nas maletas dos acadêmicos. Materiais e Métodos: Para tanto, foram analisadas 80 amostras colhidas de maletas, sendo divididas em grupos de 20, de quatro universidades, uma estadual e três particulares. O material coletado foi isolado nos meios de cultura, Ágar MacConkey e Ágar sangue com azida sódica. Resultados: Nos resultados foram identificados, cocos gram-positivos, beta hemolítico, alfa hemolítico e gama hemolítico, em sete amostras; bacilos gram- negativos, enterobactérias, em cinco amostras e coliformes, em três amostras. Conclusão: Em todas as maletas odontológicas acadêmicas foram identificadas presença de microrganismos e devem ser adequadamente desinfetadas para não disseminarem, sobretudo os fecais e patogênicos.

DESCRITORES: Exposição a agentes biológicos • Infecção • Contaminação de equipamentos
\end{abstract}

\section{ABSTRACT}

Introduction: Much has been enhanced in order to provide greater level of safety for our patients and their staff to promote health, costs of biosecurity protocols, increasingly large, reliable and hard for such an arsenal of features are employed to maximize the microbial control. Accordingly, the courses in dentistry apply heavy investments in systems that allow such control, the continued assertion of knowledge, training that puts into practice the protective barriers, which allows it to offer services free of risk, and consequently develop responsibility in disease prevention at all levels of care under the close monitoring of these attitudes on the part of educators. However, still perceived neglect of the consumption material packed in suitcases with plastic partitions, carried by the student. This study aimed to investigate the microorganisms present in the suitcases of academics. Matherials and Method: Thus, we analyzed 80 samples of suitcases, being divided into 20 groups of four universities, one state and three private. The material was isolated on culture media, MacConkey agar and blood with sodium azide. Results: The results identified, gram-positive cocci, beta hemolytic, alpha and gamma hemolytic hemolytic in seven samples, gram negative bacilli, enterobacteria, coliforms, and in five samples, three samples. Conclusion: In all the suitcases dental students were identified presence of microorganisms and must be properly disinfected to avoid spreading, particularly coliforms and pathogens

DESCRIPTORS: Exposure to biological agents • Infection • Equipment contamination.

* Professor Assistente da Disciplina de Endodontia do Curso de Odontologia da Universidade São Francisco e da Universidade Camilo Castelo Branco

****** Estagiária do Programa de Graduação do Curso de Odontologia da Universidade Camilo Castelo Branco

*** Professor da Disciplina de Periodontia do Curso de Odontologia da Universidade Cruzeiro do Sul

**** Professor da Disciplina de Periodontia do Curso de Odontologia da Universidade Cruzeiro do Sul

***** Professora do Programa de Pós-Graduação do Curso de Odontologia da Universidade Cruzeiro do Sul

****** Professor do Programa de Pós-Graduação e Graduação da Universidade de Taubaté. Disciplina de Endodontia do Departamento de Odontologia da Universidade de Taubaté 


\section{N T RO DUÇÃO}

Biossegurança é um conjunto de ações voltadas para a prevenção, minimização ou eliminação de riscos inerentes às atividades de pesquisa, produção, ensino, desenvolvimento, tecnologia e prestação de serviço, visando à saúde do homem, dos animais, a preservação do meio ambiente e a qualidade dos resultados (Fiocruz ${ }^{1}$, 2005).

De acordo com as normas de Biossegurança do Conselho Federal de Odontologia $\left(\mathrm{CFO}^{2}, 1999\right)$ os procedimentos do processamento de artigos constam, entre outros: todo instrumental reutilizável empregado nos serviços de saúde deve ser rigorosamente limpo e desinfetado ou esterilizado antes do uso em cada paciente, conforme instruções contidas neste regulamento, o processamento dos artigos e superfícies deve seguir uma sequência de passos visando o seu pleno aproveitamento, dependendo da natureza do material e da maneira como é utilizado, garantindo-se a qualidade para o reuso e a segurança dos trabalhadores envolvidos e a sequência de passos no reprocessamento deve ser a descontaminação (opcional), limpeza (opcional), enxágue (após a limpeza e/ ou descontaminação), secagem (para evitar a umidade), armazenagem (de acordo com a natureza do produto), esterilização (artigos críticos) ou desinfecção (artigos semi-críticos) e armazenagem (produtos submetidos à desinfecção ou esterilização). A estocagem pode ser feita após a realização dos passos descrita acima, de acordo com a natureza do artigo (se não-críticos) ou então após a realização das outras etapas do processamento. Deve-se utilizar área separada, limpa, livre de poeiras, em armários fechados. Os artigos esterilizados por meio físico podem ser estocados até uma semana em prateleira aberta ou até um mês se colocados sob cobertura plástica ou bolsa selada.

Esclareça-se que o princípio de biossegurança é uma questão de consciência profissional. Os procedimentos devem ser executados como um ritual, independentemente de quem seja o paciente, já que não seria ético nem suficiente submeter os pacientes a exames laboratoriais. As nor- mas de controle de infecção no consultório odontológico devem ser consideradas, pois seu entendimento é fácil, o custo é baixo e exige apenas o envolvimento do profissional e de sua equipe para alcançar resultados positivos. Se todos os profissionais se preocupassem com essas normas, a possibilidade de exposição dos pacientes a perigos seria muito reduzida (Fer$\left.\operatorname{rari}^{3}, 2001\right)$.

A proposta é analisar a ação de quatro desinfetantes utilizados em odontologia: álcool etílico a $77^{\circ} \mathrm{GL}$, composto fenólico (Duplofen), iodóforo (PVP-I) e solução de álcool etílico a $77^{\circ} \mathrm{GL}$ com $5 \%$ de clorexidina para desinfecção de superfície em quatro pontos em cada equipamento (carter, pia de lavagem de mãos, encosto de cabeça da cadeira e superfície frontal externa do refletor), utilizando-se a técnica de .spray-wipe-spray.. De cada ponto, foram coletadas amostras utilizando-se placas de superfície contendo ágar Mitis Salivarius bacitracina sacarose, ágar Sabouraud Dextrose com cloranfenicol, ágar MacConkey e ágar-sangue para contagem de estreptococos do grupo mutans, leveduras do gênero Candida, bactérias gram-negativas e contagem total de microrganismos, respectivamente (ufc/placa). Os resultados foram analisados estatisticamente utilizando-se teste $t$ de Student para comparação entre as médias de ufc/ placa. O desinfetante que demonstrou ser mais efetivo na redução microbiana foi a solução alcoólica de clorexidina, principalmente para bactérias gram-positivas. $\mathrm{O}$ iodo e o composto fenólico mostraram ser bastante eficazes na redução microbiana. O álcool etílico a $77^{\circ} \mathrm{GL}$ foi o menos eficaz dos quatro desinfetantes analisados, mas apesar de não ser indicado como desinfetante de superfície, mostrou, no presente trabalho, redução microbiana estatisticamente significativa após o processo de desinfecção (Silva e Jorge ${ }^{4}, 2002$ ).

Realizou-se a análise valendo-se da literatura a respeito, do Protocolo de Controle de Infecção no consultório odontológico, considerando-se custo e tempo que se leva na preparação do consultório para o atendimento de cada paciente. A contaminação microbiana representa verdadeira ameaça no ambiente odontológico,
HADDAD FILHO MS

LEAL TP

BELASALMA LFF

BACCI JE

SANTOS MTBR

MEDEIROS JMF

ANÁLISE DA

PRESENÇA DE

MICRORGANISMOS

DO INTERIOR

DE MALETAS

TRANSPORTADAS

por alunOS DE

GRADUAÇÃO

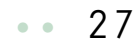

REV, ODONTOL.

UNIV, CID, SÃo

PAULO

$2012 ; 24(1)$ : 26 -

34, JAN-ABR 
HADDAD FILHO MS : LEAL TP

BELASALMA LFF BACCI JE

SANTOS MTBR

MEDEIROS JMF

ANÁLISE DA

PRESENÇA DE

MICRORGANISMOS

DO INTERIOR

DE MALETAS

TRANSPORTADAS

POR ALUNOS DE GRADUAÇÃO

28

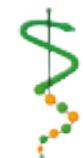

REV. ODONTOL.

UNIV. CID. SÃO

PAULO

$2012 ; 24(1): 26-$

34, JAN-ABR visto que muitas doenças como a AIDS, hepatite $\mathrm{B}$, herpes, pneumonia, tuberculose, entre outras, são transmitidas através da saliva, sangue, secreções bucais e aerossóis contaminados, nos locais em que as medidas de biossegurança são ignoradas. Portanto, é de extrema importância a realização das normas de prevenção de contaminação, a fim de assegurar a saúde dos profissionais da área odontológica e dos usuários de seus serviços. O protocolo de biossegurança é de fácil entendimento, custo baixo, necessita de um tempo mínimo para sua execução e é adequado. Assim, deve-se seguir as normas do controle de infecção, para conseguirmos reduzir significativamente o risco de contrair doenças no consultório odontológico e implantar o protocolo de biossegurança no consultório e clínica escola de Odontologia, a fim de controlar a transmissibilidade e a exposição dos pacientes a microrganismos patogênicos, minimizando os riscos de contaminação do cirurgião-dentista, da equipe auxiliar, do paciente e de pessoas de convívio rotineiro, tornando a Odontologia eficaz e segura (Pinto e Paula $\left.{ }^{5}, 2003\right)$.

A prevenção de infecção cruzada nos consultórios odontológicos e nos laboratórios de prótese dentária constitui grande desafio para os profissionais da área. Visto que a aplicação de medidas de biossegurança em laboratório de prótese é de responsabilidade do protético, o objetivo deste trabalho foi avaliar as condutas de biossegurança aplicadas nesses estabelecimentos. Os autores avaliaram 30 laboratórios de prótese das cidades de São José dos Campos e Jacareí por meio de um questionário, contendo perguntas referentes ao conhecimento dos princípios de biossegurança pelos protéticos, desinfecção dos materiais e bancadas de trabaIho e uso de equipamentos de proteção individual. Os resultados revelaram que $70,8 \%$ dos protéticos não acreditavam na possibilidade de infecção cruzada entre laboratórios de prótese e consultórios odontológicos. As substâncias utilizadas para limpeza e desinfecção das bancadas de trabalho são utilizadas sem nenhum conhecimento da eficácia desses produtos. A partir dos dados, observou-se a necessidade de alertar esses profissionais para o risco de ocorrência de infecção cruzada e a obrigatoriedade da aplicação das normas de biossegurança (Majewski et al. $\left.{ }^{6}, 2004\right)$.

Conquanto com todos os cuidados recomendados por diferentes leis, portarias, resoluções e normas técnicas do Ministério da Saúde, Ministério do Trabalho e Secretarias Estaduais e Municipais, todas as demandas relacionadas à Biossegurança, em geral, não são de maneira satisfatória adotadas pelos profissionais da área da saúde. As razões disso incluem negligência profissional ou a falta de conhecimento técnico-científico aceitável (Carmo e Costa7 , 2001, Garbin et al. ${ }^{8}$, 2005).

Quanto à terminologia, a expressão 'artigos na área de biossegurança' aplica-se aos instrumentos de diversas naturezas que podem ser veículos de contaminação. Limpeza e desinfecção de pisos, superfícies e equipamentos são obrigatórias e dentre eles cumpre destacar pontas (alta e baixa rotação, seringa tríplice, aparelho ultrassom, fotopolimerizador, cujo procedimento de limpeza é feito com água e sabão neutro, autoclave e álcool etílico 70\% a cada atendimento. Mais, ainda, material plástico, como cotonetes (limpeza com água e sabão neutro e fricção álcool $70 \%$ ) após uso, instrumental de fibra ou plástico, discos, rodas, pontas, taças de borracha (glutaraldeído) após uso, recipientes de vidro ou caixas plásticas (limpeza com detergente neutro) a cada 7 dias (PUCRS ${ }^{9}$, 2006).

Os principais equipamentos utilizados nos serviços de Odontologia são: apareIho de microabrasão; amalgamador; aparelho a laser; aparelho de radiografia intra/ extra oral; aparelho de sucção de alta/baixa potência; aparelho de ultrassom para limpeza de materiais; aparelho de polimerização de resinas; aparelho de teste elétrico de polpa dentária; aquecedor endodôntico para guta-percha; articulador de prótese dentária; esterilizadores - estufa e autoclave; unidade eletrocirúrgica; câmera intra/extra oral; contra-ângulo redutor; delineador de uso odontológico; equipamento para profilaxia odontológica bicarbonato de sódio/ultrassom. Atualmente, a prática clínica com pacientes oferece 
maior nível de segurança a estes e a toda equipe promotora da saúde, à custa de protocolos de biossegurança, e, para tal, os cursos de odontologia aplicam altos investimentos em recursos que possibilitem o controle microbiano, permitindo oferecer atendimento livre de riscos, e, consequentemente, desenvolver responsabilidade na prevenção das doenças em todos os níveis de atenção, sob o monitoramento atento dessas atitudes por parte dos educadores (Brasil ${ }^{10}, 2006$ ).

A manutenção preventiva é um programa de controle dos equipamentos. É um cuidado efetuado segundo critérios predeterminados, com a intenção de se reduzir a probabilidade de falha. É do interesse do serviço de Odontologia ter um programa de manutenção preventiva de seus equipamentos, com vistas a diminuir interrupções e perda de tempo com resultados de problemas técnicos e operacionais, tornar o equipamento amplamente disponível e confiável, conservar o seu valor e assegurar a diminuição de riscos à saúde e à vida dos pacientes. A seguir, apresentam-se algumas sugestões simples para a implantação de um programa de manutenção preventiva em equipamentos odontológicos. Essa manutenção deverá ser fundamentada no histórico de falhas do equipamento, no relato de acidentes, na classe de risco à vida do paciente, nas recomendações do fabricante e nas normas técnicas de segurança e de qualidade. Portanto, além dos testes específicos de cada equipamento, serão feitos os testes de verificações, os de segurança elétrica e as calibrações. Recomenda-se que a manutenção preventiva seja realizada em períodos em que o equipamento tenha pouco uso, não interferindo na rotina do serviço. Cada tipo de equipamento deverá ter roteiros de procedimentos diferentes e, consequentemente, um registro em forma de verificação para otimização do tempo de quem fará a intervenção preventiva (Brasil $\left.{ }^{10}, 2006\right)$.

Os profissionais de Odontologia, $\mathrm{Ci}$ rurgiões-dentistas, Auxiliares de Consultório Odontológico, Higienistas, Técnico de Higiene Dental e Técnico de Laboratório de Prótese estão sob risco constante de adquirir doenças no exercício de suas funções. Comprovadamente, o microrganismo tem driblado as medidas de segurança adotadas na atualidade, colocando em risco profissionais e pacientes, e a falta de cuidados em relação à biossegurança tem propiciado a intensificação do ciclo de infecções cruzadas. É responsabilidade do Cirurgião-dentista a orientação e manutenção da cadeia asséptica por parte da equipe Odontológica e o cumprimento das normas de qualidade e segurança quanto ao radiodiagnóstico e descarte de resíduos gerados pelo atendimento (Odontobio ${ }^{11}$, s.d.).

O controle de infecção é constituído por recursos materiais e protocolos que agrupam as recomendações para prevenção, vigilância, diagnóstico e tratamento de infecções, visando à segurança da equipe e dos pacientes, em quaisquer situações ou local onde se prestem cuidados de saúde. A biossegurança nunca é completa quando profissionais da Saúde atendem a um paciente ou manipulam instrumentos, material biológico e superfícies contaminadas. Porém, o fato de sempre haver um risco deve ser um estímulo à nossa dedicação, e não o inverso, ou seja, uma justificativa às nossas falhas (Odontobio $^{11}$, s.d.).

As principais doenças infectocontagiosas que representam riscos em consultório odontológico podem ser causadas por vírus como Catapora, Hepatite B, Hepatite C, Conjuntivite Herpética, Herpes Simples, Herpes Zoster, Mononucleose Infecciosa, Sarampo, Rubéola, Parotidite, Gripe, Papilomavírus Humano, Citomegalovírus, HIV. Podem ser causadas por bactérias que levam à Pneumonia, Infecção por Estafilococos, Estreptococos, Pseudomonas, Klebsiella, bacilos como o da Tuberculose, e ainda os fungos, mais comumente associados à Candidíase. Os profissionais de Odontologia também devem se vacinar, embora não existam todas as vacinas para prevenção dessas doenças (Odontobio ${ }^{11}$, s.d.).

Os serviços de Odontologia necessitam cumprir as normas de biossegurança baseadas em leis, portarias e normas técnicas do Ministério da Saúde, Ministério do Trabalho e Secretarias Estaduais e Municipais, que requerem proteções
HADDAD FILHO MS

LEAL TP

BELASALMA LFF

BACCI JE

SANTOS MTBR

MEDEIROS JMF

ANÁLISE DA

PRESENÇA DE

MICRORGANISMOS

DO INTERIOR

DE MALETAS

TRANSPORTADAS

por alunos de

GRADUAÇÃO

\section{9}

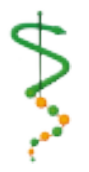

REV, ODONTOL.

UnIV. CID. São

PAULO

$2012 ; 24(1)$; 26 -

34 , JAN-ABR 
HADDAD FILHO MS :

LEAL TP

BELASALMA LFF

BACCI JE

SANTOS MTBR

MEDEIROS JMF

ANÁLISE DA

PRESENÇA DE

MICRORGANISMOS

DO INTERIOR

DE MALETAS

TRANSPORTADAS

POR ALUNOS DE GRADUAÇÃO

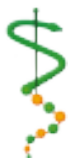

REV, ODONTOL.

UNIV, CID, SÃO

PAULO

$2012 ; 24(1): 26$

$34, J A N-A B R$

\section{ISSN 1983-5183}

contra radiações ionizantes, radiações de luz halógena, medidas para o controle de doenças infectocontagiosas, destinação de resíduos e proteção ao meio ambiente. As sanções previstas na lei podem ir desde uma simples advertência ou multa classificada em leve, grave ou gravíssima, até a interdição do estabelecimento odontológico (PortalEducação ${ }^{12}$, s.d.).

O que temos que implantar é a cultura da valorização do homem e da sua qualidade de vida. Sabemos que, a cada segundo, substâncias químicas e microrganismos estão sendo introduzidos no meio ambiente e que os resultados dessa verdadeira alquimia biotecnológica ainda são desconhecidos para a humanidade (Odontobio ${ }^{11}$, s.d.).

Ocasiões existem nas quais devem ser tratados os métodos de controle de microrganismos utilizados pelo cirurgião- dentista na clínica odontológica diária. Para prevenção da infecção cruzada na clinica odontológica, o profissional deve empregar processos de esterilização dos materiais e seguir rigorosamente todos os procedimentos destinados a manter a cadeia asséptica. Tais procedimentos são realizados em relação ao pessoal odontológico, aos instrumentos e acessórios, ao equipamento e ao paciente (Jorge ${ }^{13}$, s.d.).

Krieger et al. ${ }^{14}$ (2010) revisaram a literatura atual sobre os tópicos relacionados aos métodos de prevenção e de controle de infecção em Odontologia, cujo conhecimento e importância visam à manutenção do bem-estar daqueles que trabalham na área odontológica e dos que acessam o ambiente de prestação desse serviço. Asseveram os aludidos autores que a equipe de profissionais de Odontologia está sujeita a diversas formas de contaminação por agentes patogênicos presentes em materiais biológicos, como sangue e saliva, que são manipulados constantemente pela mesma. Esses agentes podem causar uma série de doenças infectocontagiosas graves e ainda permitir a ocorrência de um ciclo de infecção cruzada dentro e fora do ambiente odontológico, onde os profissionais, acadêmicos de Odontologia e os pacientes se tornam disseminadores de doenças. O ambiente odontológico é considerado potencialmente infecto em decorrência da presença de fluidos biológicos como saliva, sangue e coleções purulentas. Assim, os profissionais que trabalham nessa área estão sujeitos a uma série de doenças. Com o intuito de se evitar a disseminação e a propagação dessas doenças é que devem ser consideradas as medidas de Biossegurança em Odontologia, compostas por um conjunto de ações que visam à proteção do cirurgião-dentista, sua equipe e seus pacientes. A revisão feita evidenciou as medidas de combate à infecção cruzada e quebra de seu ciclo de contaminação, com vistas à promoção de um maior cuidado com a saúde.

Tão somente maletas e caixas de fibra ou plástico permitem a desinfecção e poderão ser usadas nas clínicas, sendo proibido o uso de malas revestidas de tecido para transporte de material para as clínicas (Silva et al. ${ }^{15}$, s.d.).

Entretanto, ainda se percebe negligência quanto ao material de consumo acondicionado em maletas plásticas com divisórias, transportadas pelo aluno. $\mathrm{O}$ propósito deste trabalho é investigar a presença de microrganismos nas maletas pertencentes a alunos de graduação de quatro instituições de Odontologia no Estado de São Paulo.

\section{MATERIAL E MÉTODOS}

Foram selecionadas e analisadas aleatoriamente 80 maletas utilizadas como transporte de materiais por alunos de graduação, divididas em grupos de 20, de quatro faculdades de Odontologia do Estado de São Paulo, uma estadual e três particulares. O referido projeto foi materializado e encaminhado ao Comitê de Ética em Pesquisa cujo protocolo (CEP/ UNICASTELO: 3285-3505/10) foi devidamente aprovado.

A coleta do interior das maletas plásticas foi promovida com $S$ wab que consiste de uma haste longa de polipropileno com algodão envolto em uma ou nas duas extremidades, esterilizado, umedecido em caldo BHI (Brain Heart Infusion - meio derivado da infusão de nutrientes de cérebro e coração, mais a presença de peptona como fonte de nitrogênio, carbono, 


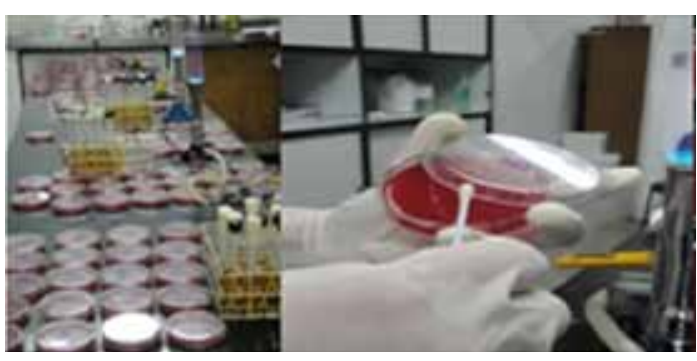

Figura 1 - Material coletado

enxofre e vitaminas, além da dextrose, constituída de carboidratos que os microrganismos utilizam para fermentação) de coloração original amarelo-claro, límpido e acondicionado em tubos de ensaio contendo a mesma substância, transportados em caixa térmica com gelo (Figura 1). As amostras foram submetidas ao período de incubação de 24 horas em estufa a $37^{\circ} \mathrm{C}$ e, a partir daí, inoculadas nos meios de cultura Ágar MacConkey e Ágar sangue com azida sódica, mantendo-se por mais 24 horas sob a mesma temperatura (Figura 2).

\section{RESULTADOS}

Nas placas com Agar sangue, cuja base nutritiva promove rápido crescimento de microrganismos, foram identificados cocos Gram-positivos (beta hemolíticos, alfa hemolíticos e gama hemolíticos) em todas as oitenta amostras. A azida sódica presente nesse meio inibiu o crescimento de bacilo Gram-negativo (Figura 3).

Nas placas com Agar MacConkey, meio diferencial para cultivo e isolamento

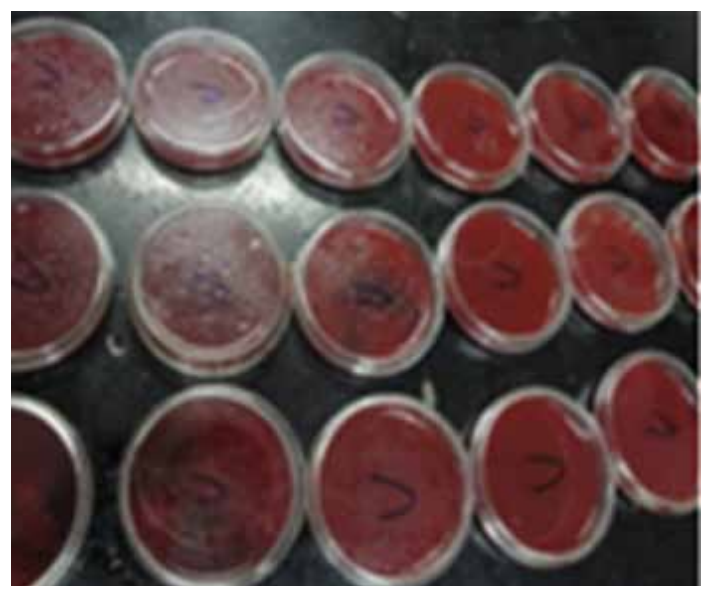

Figura 2 - Período de incubação de 24 horas

de E. Coli, composto de peptona, foram observados bacilos Gram-negativos, bactérias que fermentam lactose (coliformes), em duas amostras, e que não fermentam lactose (enterobactérias) em seis amostras (Figura 4).

\section{I SCUSSÃO}

O transporte de maletas por estudantes de Odontologia é uma tarefa costumeira no dia a dia, cuja finalidade é levar material para sua bancada de trabalho na instituição onde estudam. Dentre outros equipamentos e materiais levados no interior dessas maletas, cumpre citar caixa de cones de guta-percha, anestésicos, medicação intracanal em tubetes, vidros ou frascos contendo EDTA-T e cimentos de selamento temporário, lençol de borracha para isolamento absoluto e sugadores.

Ora, as superfícies desses materiais e

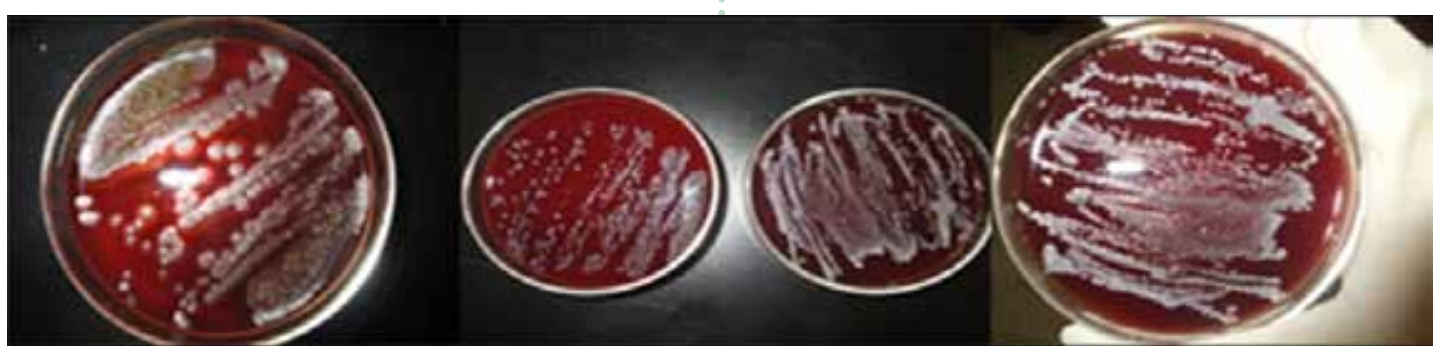

Figura 3 - Placas de Agar sangue para identificação das amostras
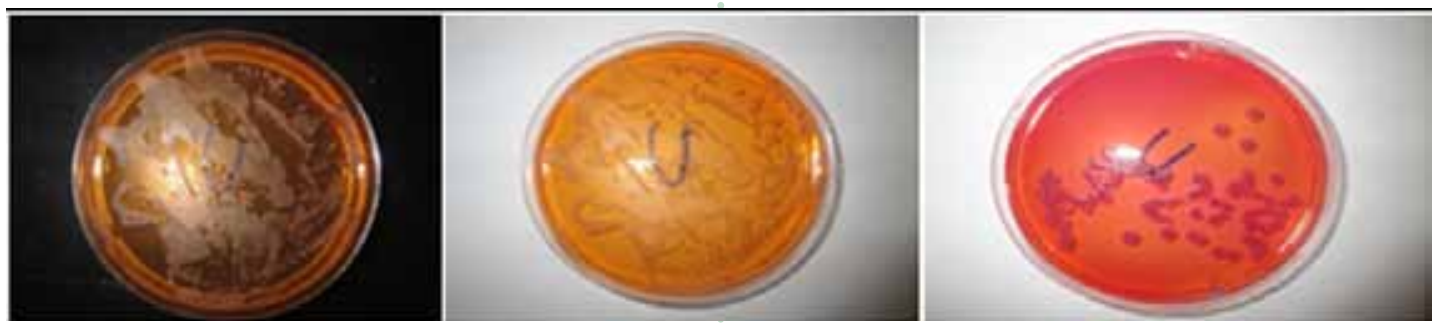

Figura 4 - Placas com Agar Mac Conkey

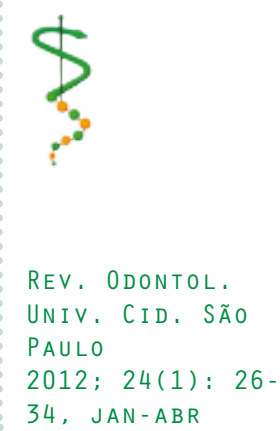


HADDAD FILHO MS : LEAL TP

BELASALMA LFF BACCI JE

SANTOS MTBR

MEDEIROS JMF

ANÁLISE DA

PRESENÇA DE

MICRORGANISMOS

DO INTERIOR

DE MALETAS

TRANSPORTADAS

POR ALUNOS DE GRADUAÇÃO

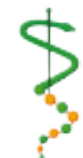

REV, ODONTOL

UNIV. CID. SÃO

PAULO

$2012 ; 24(1): 26$ -

34, JAN-ABR
I SSN 1983-5183

equipamentos evidentemente são ou já estão contaminados por agentes microbianos, constituindo verdadeiros veículos de bactérias que adentrarão para as clínicas onde estão pacientes, alunos, professores e funcionários da área de saúde, sem contar de que maneira são transportados, o ambiente que os rodeia e como são acondicionados no interior dessas maletas.

Tal acontecimento é verdadeiro quando se observou que todas as 80 amostras que foram colhidas de tais maletas estavam contaminadas, nelas sendo identificados cocos Gram-positivos (beta hemolíticos, alfa hemolíticos e gama hemolíticos), sendo que a azida sódica presente nesse meio inibiu o crescimento do bacilo Gram-negativo.

Do mesmo modo, foram detectados bacilos Gram-negativos, bactérias que fermentam lactose (coliformes) em duas amostras e aquelas que não fermentam lactose (enterobactérias) em seis amostras.

Isso posto, entende-se que há necessidade de cuidados com relação a qualquer tipo de equipamento e material levados para dentro da clínica, sendo extremamente necessária a desinfecção de suas superfícies para que estas fiquem isentas de bactérias.

Talvez não haja como realizar a descontaminação do interior das maletas, todavia, todo e qualquer material ou equipamento transportados, antes de depositá-los nas mesas auxiliares da clínica, estes sim precisam ser desinfetados.

Aliás, Silva et al. ${ }^{15}$ apontam que as maletas permitem desinfecção desde que sejam de fibra ou plástico, não sendo possível em malas revestidas de tecido, vedadas para o transporte de material para a clínica.

Segundo a Agência Nacional de Vigilância Sanitária, Brasil ${ }^{10}$ (2006), a classificação dos organismos segundo seu potencial patogênico indica que os encontrados nas maletas são considerados risco classe 2, ou seja, risco individual moderado de patógenos que causam infecções; entretanto, há medidas eficazes na prevenção; e risco classe 3 de patógenos que representam maior agressividade a quem os manipula e ainda maior aos pacientes que têm contato direto e invasivo com esses agentes, a exemplo de um tratamento endodôntico ou cirúrgico, cujo risco é abortado quando da utilização de medidas eficazes de prevenção, o que não se percebeu nesta investigação.

A recomendação do Ministério da Saúde ${ }^{10}$ sinaliza que as superfícies devem ser desinfetadas no final do procedimento e, para tal, recomenda-se proteção de luva doméstica não descartável e, após o uso, lavar as mãos enluvadas com água e sabão e descontaminá-las em solução de hipoclorito de sódio a 0,5\% durante trinta a sessenta minutos.

Ainda que protocolos, manuais, guia de biossegurança disseminem informações e cuidados, aliados ao monitoramento dos educadores, notou-se que a maioria dos acadêmicos observados utilizava sobreluvas quando da manipulação das maletas como forma de não contaminá-las; no entanto, não atentam para o fato desse ambiente ser contaminado, e mais, em nenhum momento foi registrado esvaziamento das mesmas ao final do atendimento e desinfecção da superfície plástica interna e externa e de todos os materiais individualmente acondicionados. Tanto pior que, além do contato e do transporte do microrganismo, o operador também pode ser veículo da contaminação química em decorrência de tantos produtos que são acondicionados em tubetes de $1,8 \mathrm{ml}$, como anestésicos, medicações intracanais e substâncias químicas auxiliares.

Com relação a isso, Silva Júnior et al. ${ }^{16}$ (1991) realizaram estudo sobre a evidenciação de microrganismos em tubetes anestésicos não usados e constataram presença de bacilos gram-negativos, estafilococos gram-positivos e fungos em 25 das 38 amostras analisadas. Tal fato, segundo os autores, pode advir da contaminação prévia das tampas de borracha e metal decorrente da indevida estocagem e transporte inadequado, e que a própria agulha, ao perfurar a tampa de borracha, possibilitaria a passagem de microrganismos para o produto que, quando aplicado, transmite infecções.

Pode-se presumir, quanto a essa preocupante constatação de microrganismos nas maletas, que o ciclo, por vezes, tem seu início a partir da estocagem no comér- 
cio, ou seja, o acadêmico acomodaria o material ou embalagem na maleta já contaminada, fato ainda somado ao transporte descuidado, exposição ao calor e, fundamentalmente, a manipulação sem cautela de proteção, ausência de descontaminação prévia, manipulação sem sobreluvas, contato no interior da maleta por outros alunos quando do empréstimo de materiais, enfim, hábitos que, se devidamente conscientizados pelo graduando, minimizariam a disseminação de agentes nocivos às custas de produtos como álcool, hipoclorito de sódio, desinfetantes fenólicos, entre outros.

Estudos realizados em diferentes estados verificaram a tendência dos acadêmicos em burlar as normas de biossegurança (Cardoso $\left.{ }^{17}, 1997\right)$.

Nesse sentido, Medeiros et al. ${ }^{18}$ (1998), analisando comportamento de alunos de último período de seis faculdades do Rio de Janeiro, constataram que os mesmos não seguem corretamente as normas de biossegurança e que o índice de acerto observado foi de apenas $60 \%$.

O que temos que implantar é a cultura da valorização do homem e a valorização da sua qualidade de vida. Sabemos que, a cada segundo, substâncias químicas e microrganismos estão sendo introduzidos no meio ambiente e que os resultados dessa verdadeira alquimia biotecnológica ainda são desconhecidos para a humanidade (Odontobio ${ }^{11}$, s.d.).

O cirurgião-dentista tem que adotar as medidas preventivas em todos os pacientes, sem exceções. Assim, seguindo-se as normas de biossegurança, o risco de se contrair doenças em um ambiente odontológico se reduz significativamente tanto para os profissionais quanto para os pacientes (Pinto e Paula ${ }^{5}$, 2003).

Desinfecção de superfície são procedimentos realizados nas áreas externas do equipamento odontológico e demais itens do consultório (Silva e Jorge ${ }^{4}, 2002$ ).

Nesse sentido, registra-se que nesta investigação foi avaliada a ação de nanopartículas de prata como recurso mais eficaz e prático na descontaminação dessa superfície e que, oportunamente, os resultados serão apresentados.

\section{CONCLUSÕES}

Em todas as maletas utilizadas por acadêmicos para acondicionamento e transporte de materiais odontológicos identificou-se presença de microrganismos.

Nas placas com Ágar sangue foram identificados cocos gram-positivo (beta hemolíticos, alfa hemolíticos e gama hemolíticos) em todas as oitenta amostras. A azida sódica presente nesse meio inibiu o crescimento de bacilo gram-negativo.

Nas placas com Ágar MacConkey observaram-se bacilos gram-negativos, bactérias que fermentam lactose (coliformes) em duas amostras e que não fermentam lactose (enterobactérias) em seis amostras.

As maletas devem ser adequadamente desinfetadas para não disseminarem microrganismos, sobretudo os fecais e patogênicos.

\section{AGRADECIMENTOS}

Agradecemos à Técnica de Laboratório Informática Renata Maria Monteiro Moreira do Departamento de Engenharia Mecânica da Universidade de Taubaté pelo apoio na elaboração das fotografias.

\section{REFERÊNCIAS}

1. FIOCRUZ. Procedimentos para a manipulação de microorganismos patogênicos e/ ou recombinantes na FIOCRUZ. 2005; [acesso em 25 de ago. 2010] Available from: http://www.biossegurancahospitalar.com.br/files/livroprocedmanipmicropato.pdf.

2. CFO CFdO. Biossegurança. 1999; [acesso em 25 de ago. 2010] Available from: http://cfo.org.br/wp-content/uploads/2009/09/manual_biosseguranca.pdf.

3. Ferrari P. Princípio de Biossegurança é uma questão de consciência profissional. Revista Interativo 2001 jun./ago.; VI(48):

HADDAD FILHO MS

LEAL TP

BELASALMA LFF

BACCI JE

SANTOS MTBR

MEDEIROS JMF

ANÁLISE DA

PRESENÇA DE

MICRORGANISMOS

DO INTERIOR

DE MALETAS

tRANSPORTADAS

por alunos de

GRADUAÇÃO

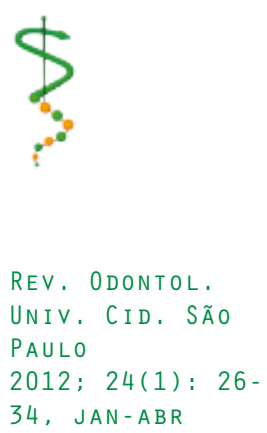


HADDAD FILHO MS LEAL TP BELASALMA LFF BACCI JE SANTOS MTBR MEDEIROS JMF

ANÁLISE DA PRESENÇA DE MICRORGANISMOS DO INTERIOR DE MALETAS TRANSPORTADAS POR ALUNOS DE GRADUAÇÃO

REV, ODONTOL,

UNIV. CID. SÃO PAULO $2012 ; 24(1): 26-$ 34, JAN-ABR:
4. Silva CRGe, Jorge AOC. Avaliação de desinfetantes de superfície utilizados em Odontologia. Pesquisa Odontológica Brasileira 2002 16(107-14.

5. Pinto K, Paula C. Protocolo de biossegurança no consultório odontológico: custo e tempo. Rev Biociênc 2003 9(4):19-23.

6. Majewski M, Koga-Ito C, Junqueira J, Jorge A. Avaliação das condutas de biossegurança aplicadas em laboratórios de prótese dentária. Rev Biociência 2004 10(3):1616.

7. Carmo M, Costa A. Procedimentos de biossegurança em Odontologia. JBC J Bras Clin Estet Odontol 2001 mar.-abr.;5(26):116-9.

8. Garbin AJ, Garbin CA, Arcieri RM, Crossato M, Ferreira NF. Biosecurity in public and private office. J App/ Oral Sci 2005 Jun;13(2):163-6.

9. PUCRS. Manual de biossegurança dos ambulatórios da Faculdade de Odontologia da PUCRS. 2006; [acesso em 25 de ago. 2010] Available from: http://www.pucrs. br/odonto/manual.pdf.

10. Brasil MdSANdVS. Serviços Odontológicos: prevenção e controle de riscos. Brasília: Ministério da Saúde; [acesso em 30 de ago. 2010] 2006. Available from: http:// www.opas.org.br/gentequefazsaude/bvsde/bvsacd/cd49/manual_odonto.pdf.

11. Odontobio. Odontologia e biossegurança. s.d.; [acesso em 30 de ago. 2010] Available from: http://www.odontobio.kit.net/manual.htm.

12. PortalEducação. Lei federal n0 6.437, de 20/08/1977. s.d.; [acesso em 24 de out. 2010] Available from: http://www.portaleducacao.com.br/odontologia/artigos/3066/lei-federal-n-6437-de-20-08-1977.

13. Jorge A. Princípio de biossegurança em odontologia. s.d.; [acesso em 24 de out. 2010] Available from: http://www.higieneocupacional.com.br/download/biosseg-odonto.pdf.

14. Krieger D, Bueno R, Gabardo M. Perspectivas de biossegurança em odontologia. Revista Gestão \& Saúde 2010 1(0):1-10.

15. Silva A, Flório F, Ramacciato J, Cury P, Motta R, Teixeira R. Protocolo de Biossegurança. s.d.; [acesso em 24 de out. 2010] Available from: http://www.slmandic.com. br/download/protocolo-de-biosseguranca-2008.pdf.

16. Silva Júnior M, Seixas A, Seixas A. Tubetes anestésicos: uma avaliação microbiológica. Rev Ass Paul Cir Dent 1991 jan.-fev.;45(1):367-8.

17. Cardoso A. Uso das normas de controle de infecções pelos estudantes de odontologia de seis Faculdades do Rio de Janeiro [Monografia de Graduação]. Rio de Janeiro: Universidade Federal do Rio de Janeiro; 1997.

18. Medeiros V, Cardoso A, Ferreira S. Uso das normas de controle de infecção na prática odontológica. Rev Bras Odontol 1998 jul.-ago.;55(4):209-15.

Recebido em: 01/12/2011

Aceito em: 09/04/2012 